\title{
Optimization of the New Index Reaching Law of the Active Suspension Sliding Mode Controller Based on the Cuckoo Search Algorithm
}

\author{
Shouwei Wei and Xiaoyu Su 10 \\ School of Electrical and Electronic Engineering, Shanghai University of Engineering Science, Shanghai, China \\ Correspondence should be addressed to Xiaoyu Su; su_xy0722@163.com
}

Received 2 February 2021; Revised 21 September 2021; Accepted 27 September 2021; Published 26 October 2021

Academic Editor: Weixiang Zhou

Copyright () 2021 Shouwei Wei and Xiaoyu Su. This is an open access article distributed under the Creative Commons Attribution License, which permits unrestricted use, distribution, and reproduction in any medium, provided the original work is properly cited.

To facilitate the performance of the active suspension system, the optimization of a new reaching law of the active suspension sliding mode controller based on cuckoo algorithm is addressed in this paper. Firstly, a linear model of the active suspension system is built. Then, according to the features of the new exponential reaching law, an active sliding mode control scheme based on the new sliding mode reaching law is designed. Finally, the simulation results are separated into two stages to verify the suitability and superiority of the proposed control scenario.

\section{Introduction}

Because of the rapid development of the manufacturing industry, the realization of enhancement for both quantity and quality of products has been changed from a simple realization to a more accurate and intelligent realization. Specifically, China's car manufacturing has faced with higher demand in recent years. For instance, to get more smoothness and comfortless by improving the active suspension system's performance indexes, various excellent and efficient control schemes for the active suspension system have been proposed in the field of automobile control [1-7]. Pan and Sun designed an output feedback finite-time control method to solve the problem of how to stabilize the perturbed vehicle active suspension system. A disturbance compensator with finitetime convergence performance is proposed to compensate the unknown external disturbance [2]. In their later studies, Pan et al. also proposed an adaptive fault-tolerant method to synthesize compensation controllers for nonlinear active suspension systems with dead-zone actuators and random faults. An actuator fault compensation scheme is proposed, which can achieve arbitrarily small tracking errors in the presence of nonlinear actuators with random faults [5]. And there are more and more control strategies for active suspension, such as Ho control [4, 8-11], optimal control [12-15], neural network control [16-19], LQG control [20, 21], predictive control [22], and sliding mode control [14, 23].

Sliding mode control has many advantages, such as fast response, insensitivity to disturbance, and no need for an online recognition system. Many scholars have studied sliding mode control schemes and applications [24, 25]. Jiang et al. focused on the event-triggered fuzzy sliding mode control of a networked control system with semi-Markov process control. The reachability of the predefined switch surface and the ideal sliding motion of the fuzzy sliding mode controller are established. The numerical simulation of the single-link robot arm system verifies the applicability of the proposed research results [24]. Zhen et al. not only proposed a novel linear sliding surface based on the observer and output information but also designed the corresponding sliding mode control law to ensure that the sliding surface almost reaches the target by the adaptive method [25]. Some scholars design the sliding mode controller according to the active suspension system and make the vehicle have better performance of vehicle suspension under the action of the sliding mode controller [26-28]. In [26], the authors designed a sliding mode controller which combined two sliding modes to limit the vertical displacement of the front body and the rear body. The 
simulation results are verified under three different assumptions. In [27], Jiang and Karimi studied the sliding mode control problem of nonlinear stochastic Markov jump systems with uncertain time-varying delays, in which a fuzzy observer system was constructed to transform the system into two lower-order subsystems. On this basis, the adaptive controller is synthesized by using the information of bounded time delay. The effectiveness of the method is verified by a single-link robot arm model. Wang et al. introduced the SMC strategy into the study of discrete-time Markov jump SPSs, using this method to study the sliding mode control problem of the slow sampling singularly operating system with Markov jump parameters, which made a certain contribution to the application of sliding mode control [28]. Yang et al. studied sliding mode control for a class of discrete-time switched systems. It is a great breakthrough in the sliding mode control field in that the residence duration switching rule is considered in the research of sliding mode control [29].

The design of the sliding mode control scheme has been improved in the literature, but the parameter selection of the sliding mode controller is also essential. And different parameters will lead to a substantial gap in the performance of the controller. With the development of the optimization algorithms, this problem has been solved to a large extent. In [30], Khoudiri et al. proposed a new optimal sliding mode controller with fixed switching frequency for the boost converter. The range of parameters of the sliding mode controller is obtained by using the simplex method, and then the particle swarm optimization algorithm (PSO) is used to find the parameters that make the controller obtain the best performance. In [31], Sun selected the output function of the controller and the system following error function as the fitness function of the particle swarm optimization algorithm. By using the optimized parameters, the performance of the controller was significantly improved. Gao et al. designed a sliding mode controller for the 4-DOF active suspension model and optimized the parameters of the sliding mode controller with genetic algorithm so that the performance of the active suspension could be improved [32].

All the above scholars have largely optimized the parameters of the sliding mode controller. Nevertheless, traditional optimization algorithms such as particle swarm optimization (PSO) and genetic algorithm (GA) are prone to fall into the local optimal solution, and the implementation process is too complex. This paper will use the superior cuckoo algorithm to optimize the parameters of the new exponential reaching law in active suspension. Cuckoo search (CS) algorithm is favored by many scholars due to its simple structure, few control parameters, excellent search path, and strong global optimization ability. As one of the performance indexes of active suspension, the dynamic deflection of suspension reflects the severity of vibration during driving, which is closely related to the impact of the vehicle body on the ground and the driving stability of the vehicle. At the same time, considering the service life of the vehicle suspension, the fitness function of cuckoo algorithm is designed based on the dynamic deflection of suspension. In the design process of the active suspension sliding mode controller, most scholars still use the traditional reaching law design method. According to the new sliding mode reaching law proposed in [33], a sliding mode control scheme for active suspension based on the new exponential reaching law is designed in this paper. In this paper, a new exponential reaching law is proposed by adding exponential parameters $\left|x_{n}\right|^{a}$ to the original ordinary reaching law. It enables the new approach rate design method to optimize the index of $x_{n}$ more quickly and efficiently. The difficulty of this method lies in the selection of $x_{n}$. This parameter should be of sufficient importance to the control model. When optimizing this parameter, it should be able to optimize other performance indexes, so as to achieve the best effect.

To solve the above problems, a sliding mode controller parameter optimization method based on cuckoo algorithm is proposed. The main contributions of this paper are as follows: (1) a sliding mode controller based on the new reaching law is built; (2) cuckoo search algorithm is used to find the best parameters of the active suspension sliding mode controller; (3) in the analysis of simulation results, the controllers under different reaching laws and before and after algorithm optimization are compared twice. To sum up, some scholars have improved the sliding mode control method, while others have improved the parameter selection scheme. Therefore, the motivation of this paper is to optimize the parameters by using the cuckoo search algorithm with superior performance after improving the approach law of the sliding mode control scheme based on the reaching law. The sum of the two optimization methods makes the performance of the sliding mode controller reach the best.

The rest of this paper is arranged as follows: the active suspension model is established in Section 2. In Section 3, the new exponential reaching law is used to design the sliding mode controller, and the relevant parameters of the algorithm are given. The simulation results of the two schemes are given in Section 4. And the conclusion of this paper is presented in Section 5.

\section{General Linear Active Suspension Model}

In the traditional passive suspension research and design process, it is difficult to consider both smoothness and control stability. The active suspension can adjust the output force to maintain a reasonable height from the ground to improve the handling stability, stability, and trafficability of the vehicle. In this paper, the $1 / 4$ active suspension model shown in Figure 1 is selected as the control object.

The dynamic model of the system obtained in Figure 1 is as follows:

$$
\left\{\begin{array}{l}
m_{t} \ddot{x}_{t}-c_{s}\left(\dot{x}_{s}-\dot{x}_{t}\right)+k_{t}\left(x_{t}-x_{r}\right)-k_{s}\left(x_{s}-x_{t}\right)=-U \\
m_{s} \ddot{x}_{s}+c_{s}\left(\dot{x}_{s}-\dot{x}_{t}\right)+k_{s}\left(x_{s}-x_{t}\right)=U
\end{array}\right.
$$




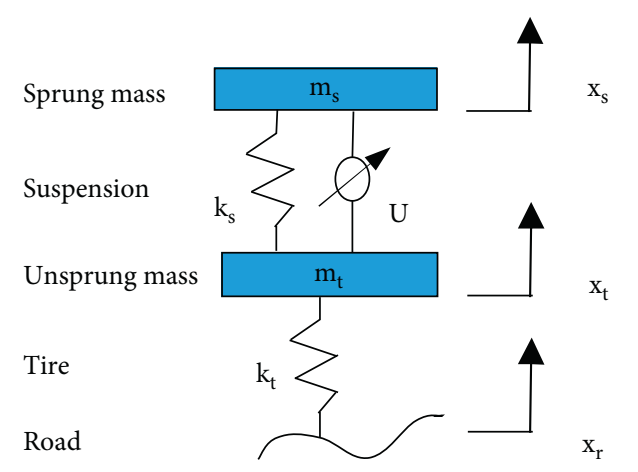

Figure 1: Suspension model.

where $m_{s}$ and $m_{t}$ denote the sprung and unsprung masses, respectively; $k_{s}$ is the stiffness of the suspension; $k_{t}$ stands for the tire stiffness; $c_{s}$ represents the damping coefficient; $x_{s}$ and $x_{t}$ are the vertical displacement of the sprung mass and the displacement of the unsprung mass, respectively; $x_{\mathrm{r}}$ is the vertical road disturbance; and $U$ indicates the actual control force. The suspension springs and tires are assumed to be linear, and the whole model is therefore linear. The selection of state-space variables is as follows:

$$
\begin{aligned}
& X=\left[x_{1}, x_{2}, x_{3}, x_{4}\right]^{T}, \\
& x_{1}=x_{t}, \\
& x_{2}=x_{s}, \\
& x_{3}=\dot{x}_{t}, \\
& x_{4}=\dot{x}_{s} .
\end{aligned}
$$

Then, the state-space expression can be expressed as follows:

$$
\dot{X}=A X+B V+G W .
$$

The matrix parameters in the state-space expression are

$$
\begin{aligned}
& A=\left[\begin{array}{cccc}
0 & 0 & 1 & 0 \\
0 & 0 & 0 & 1 \\
-\frac{k_{t}+k_{s}}{m_{t}} & \frac{k_{s}}{m_{t}} & -\frac{c_{s}}{m_{t}} & \frac{c_{s}}{m_{t}} \\
\frac{k_{s}}{m_{s}} & -\frac{k_{s}}{m_{s}} & \frac{c_{s}}{m_{s}} & -\frac{c_{s}}{m_{s}}
\end{array}\right], \\
& B=\left[\begin{array}{llll}
0 & 0 & -\frac{1}{\mathrm{~m}_{\mathrm{t}}} & \frac{1}{\mathrm{~m}_{\mathrm{s}}}
\end{array}\right]^{T}, \\
& G=\left[\begin{array}{llll}
0 & 0 & \frac{\mathrm{k}_{\mathrm{t}}}{\mathrm{m}_{\mathrm{t}}} & 0
\end{array}\right]^{T} \text {. }
\end{aligned}
$$

\section{Sliding Mode Control Design}

3.1. Sliding Mode Controller Based on the New Approach Law. Last several years, sliding mode control has attracted increasing attention due to its fast response to input and insensitive to perturbation. Sliding mode control consists of two parts: approach motion and sliding mode motion. The approach process is the normal motion stage of the system under continuous control. All its trajectories in the state space are located outside the switching surface or passing through the switching surface in a limited way. Sliding mode motion is the phase in which the system moves near the switching surface and along the switching direction towards the stable point. A new exponential approach law is adopted to reach the sliding mode surface more quickly:

$$
\begin{aligned}
\dot{S}_{N}(x) & =-\varepsilon \operatorname{sgn} S_{N}(x)-k\left|x_{n}\right|^{a} S_{N}(x), \\
\varepsilon & >0, \\
k & >0 .
\end{aligned}
$$

Compare the above formula (5) with

$$
\dot{S}_{s}(x)=-\varepsilon_{s} \operatorname{sgn} S(x)-k_{s} S(x) \text {. }
$$

In the new exponential reaching law (5), the special function $\left|x_{n}\right|^{a}$ of the state variable is added, and $a$ is a constant. The control effect of the performance index of variable $\left|x_{n}\right|^{a}$ is affected by its exponential composition with the system variable $x_{n}$, where $S_{N}(x)$ and $S s(x)$ denote the new and common sliding surface, respectively; sgn is a symbolic function, when $s$ is greater than 0 , it is taken as 1 , and when $s$ is less than 0 , it is taken as -1 ; and $\varepsilon, \varepsilon_{s}, k$, and $k_{s}$ are all coefficients. Zhang et al. [33] proved that the new exponential approach law can effectively accelerate the convergence process of selected state variables.

The existence of the sliding surface makes the sliding mode control asymptotically stable and has good quality. The sliding surface also represents the ideal dynamic characteristics of the control system. The sliding surface of the active suspension is designed as follows:

$$
\left\{\begin{array}{l}
S(X)=C X, \\
C=\left[\begin{array}{llll}
c_{1} & c_{2} & c_{3} & 1
\end{array}\right],
\end{array}\right.
$$

where $c_{1}, c_{2}$, and $c_{3}$ are constants. In sliding mode control, the constants $c_{1}, c_{2}$, and $c_{3}$ must satisfy the Hurwitz criterion to make the system stable. In the design of switching hyperplane, $W$ is ignored, and it is considered added in the design of control.

The matrix transformation is performed as follows:

$$
\begin{aligned}
B & =\left[\begin{array}{l}
B_{1} \\
B_{2}
\end{array}\right], \\
B_{1} & \in R^{3}, \\
B_{2} & \in R^{1} .
\end{aligned}
$$


The transformation matrix is

$$
T=\left[\begin{array}{cc}
I_{3} & -B_{1} B_{2}^{-1} \\
0 & I_{1}
\end{array}\right]=\left[\begin{array}{cccc}
1 & 0 & 0 & 0 \\
0 & 1 & 0 & 0 \\
0 & 0 & 1 & \frac{\mathrm{m}_{\mathrm{s}}}{\mathrm{m}_{\mathrm{t}}} \\
0 & 0 & 0 & 1
\end{array}\right] .
$$

Make the following conversion:

$$
\begin{aligned}
& \tilde{X}=T X=\left[\begin{array}{c}
X_{1} \\
X_{2}
\end{array}\right]=\left[\begin{array}{c}
x_{t} \\
x_{s} \\
\dot{x}_{t}+\frac{m_{s}}{m_{t}} \dot{x}_{s} \\
\dot{x}_{s}
\end{array}\right] \text {, } \\
& \widetilde{A}=T A T^{-1}=\left[\begin{array}{ll}
\widetilde{A}_{11} & \widetilde{A}_{12} \\
\widetilde{A}_{21} & \widetilde{A}_{22}
\end{array}\right] \\
& =\left[\begin{array}{ccccc}
0 & 0 & 1 & \vdots & -\frac{m_{s}}{m_{t}} \\
0 & 0 & 0 & \vdots & 1 \\
-\frac{k_{t}}{m_{t}} & 0 & 0 & \vdots & 0 \\
\cdots & \cdots & \cdots & \vdots & \cdots \\
\frac{k_{s}}{m_{s}} & -\frac{k_{s}}{m_{s}} & \frac{c_{s}}{m_{s}} & \vdots & -c_{s}\left(\frac{m_{s}+m_{t}}{m_{s}+m_{t}}\right)
\end{array}\right]
\end{aligned}
$$$$
\widetilde{B}=T B=\left[\begin{array}{c}
0_{3 \times 1} \\
\widetilde{B}_{21}
\end{array}\right]=\left[\begin{array}{c}
0 \\
0 \\
0 \\
\frac{1}{m_{s}}
\end{array}\right],
$$$$
\widetilde{G}=T B=\left[\begin{array}{c}
\widetilde{G}_{11} \\
0
\end{array}\right]=\left[\begin{array}{c}
0 \\
0 \\
\frac{k_{t}}{m_{t}} \\
0
\end{array}\right] .
$$

The standard types can be obtained as follows:

$$
\begin{aligned}
{\left[\begin{array}{l}
\dot{X}_{1} \\
\dot{X}_{2}
\end{array}\right] } & =\left[\begin{array}{ll}
\widetilde{A}_{11} & \widetilde{A}_{12} \\
\widetilde{A}_{21} & \widetilde{A}_{22}
\end{array}\right]\left[\begin{array}{c}
X_{1} \\
X_{2}
\end{array}\right]+\left[\begin{array}{c}
0 \\
\widetilde{B}_{21}
\end{array}\right] V+\left[\begin{array}{c}
\widetilde{G}_{11} \\
0
\end{array}\right] W, \\
S(x) & =\left[\begin{array}{ll}
\widetilde{C}_{1} & \widetilde{C}_{2}
\end{array}\right]\left[\begin{array}{l}
X_{1} \\
X_{2}
\end{array}\right],
\end{aligned}
$$

where

$$
\widetilde{C}=C T^{-1}=\left[\begin{array}{ll}
\widetilde{C}_{1} & \widetilde{C}_{2}
\end{array}\right]=\left[\begin{array}{lllll}
c_{1} & c_{2} & c_{3} & \vdots & \frac{m_{t}-m_{s} c_{3}}{m_{t}}
\end{array}\right] .
$$

According to (11) and (12), the following equation can be obtained on the sliding surface:

$$
\begin{aligned}
& X_{2}=-\widetilde{C}_{2}^{-1} \widetilde{C}_{1} X_{1}, \\
& \left(\mathrm{~m}_{\mathrm{t}}-\mathrm{m}_{\mathrm{s}} \mathrm{c}_{3} \neq 0\right), \\
& \dot{X}_{1}=\left(A_{11}-A_{12} \widetilde{C}_{2}^{-1} \widetilde{C}_{1}\right) X_{1} .
\end{aligned}
$$

The characteristic polynomial of (15) is

$$
D(\lambda)=\lambda^{3}+\frac{m_{t} c_{2}+m_{s} c_{1}}{m_{t}-m_{s} c_{3}} \lambda^{2}+\frac{k_{t}}{m_{t}-m_{s} c_{3}} \lambda+\frac{k_{t} c_{1}}{m_{t}-m_{s} c_{3}} .
$$

According to the Hurwitz criterion, the following restrictions can be obtained:

$$
\left\{\begin{array}{l}
c_{2} c_{3}-c_{1}>0, \\
c_{2}\left(m_{s}-m_{t} c_{3}\right)>0, \\
c_{2}\left(m_{s} c_{2}-m_{t} c_{1}\right)>0 .
\end{array}\right.
$$

By combining sliding variable (12) with (3) and (15), the control force can be obtained as follows:

$$
U_{N}=-(C B)^{-1}\left[C A X+C G W+\varepsilon \operatorname{sgn} S(x)+k\left|x_{n}\right|^{a} S(x)\right] .
$$

For the actual vibration reduction system, since the external disturbance $W$ is difficult to be detected and estimated online, it is required to decouple the control from the external disturbance and make the coefficient of disturbance equal to 0 . The following can be obtained: $c_{1}\left\langle 0, c_{2}\right\rangle 0$, and $c_{3}=0$.

$$
U_{N}=-(C B)^{-1}\left[C A X+\varepsilon \operatorname{sgn} S(x)+k\left|x_{n}\right|^{a} S(x)\right] .
$$

Then, define the Lyapunov function:

$$
V(x)=\frac{1}{2} S^{2}
$$

The derivative of this formula can be obtained as $\dot{V}(x)=S \dot{S}$. If the system keeps $V(x)<0$ in the normal state, then Lyapunov's theory tells us that the system will eventually reach the sliding surface. 
3.2. Parameter Setting of Cuckoo Search Algorithm. Although the sliding mode control has good performance, the selection of controller parameters has a very obvious impact on the control effect. The new sliding mode approach law parameters are optimized by using the cuckoo search algorithm, and the optimal performance of the controller is obtained. The algorithm not only has few control parameters and is not controlled by complex external instructions but also can effectively deal with complex optimization problems such as single peak value and multipeak value. Moreover, when cuckoo search algorithm performs global search based on Levy flight, the value of frequent small steps and occasional big steps makes it to have rich search behavior in the solution space, and the search direction is flexible.

Cuckoo search algorithm is an efficient metaheuristic optimization algorithm proposed by Xin She Yang and Suash Deb of Cambridge University, which imitates cuckoo's special breeding and reproduction mode. In nature, cuckoos do not build their own nests. Instead, they look for other birds' nests to lay eggs in and let other birds help them hatch and reproduce. However, there is a chance that the host will find the intruder's eggs, at which point the host will choose to clear them or abandon the nest. Cuckoo search algorithm meets the following rules:

(1) Each cuckoo generation lays only one egg and randomly chooses the nest for parasitic breeding.

(2) In the randomly selected generation group, the best offspring can carry out the operation of reproducing the next generation.

(3) The number of nests $N$ remains unchanged, and the probability that the eggs are found to be foreign eggs by the host of the nest is $P a . P a \in[0,1]$.

Levy flight search method with strong randomness has been applied in cuckoo algorithm:

$$
X_{t+1}=X_{t}+\alpha \otimes \operatorname{Levy}(s),
$$

where $X_{t+1}$ and $X_{t}$ denote the nest positions of the $t+1$ and $t$ generation, respectively; $\alpha$ represents the step control quantity; $\otimes$ denotes point-to-point multiplication; $n$ is the number of bird's nests, which also represents the number of feasible solutions; Levy $(s)$ is the random search path; and $s$ is the random step size of the Levy flight. The expression is as follows:

$$
\begin{aligned}
s & =\frac{u}{|v|^{1 / \beta}}, \\
u & \sim N\left(0, \sigma^{2}\right), \\
v & \sim N(0,1), \\
\sigma & =\left\{\frac{\gamma(1+\beta) \sin (\pi \beta / 2)}{\beta \gamma((1+\beta) / 2) 2^{(\beta-1 / 2)}}\right\} .
\end{aligned}
$$

When the situation in criterion (3) occurs, regardless of the effect of the solution, it will be cancelled. For the cuckoo algorithm, a new nest will be created at this time. The updated formula for the location of the new nest is

$$
X_{\text {new }}=X_{t}+r \otimes \operatorname{Heaviside}(P a-e) \otimes\left(X_{i}-X_{j}\right),
$$

where $X_{\text {new }}$ is the newly built nest; $r$ and $e$ are the random number of uniform distributions; and $X_{i}$ and $X_{j}$ are the positions of any two nests. The optimization scheme is shown in Figure 2.

As an important index of suspension design, the dynamic deflection of suspension is of great significance. Only by ensuring the performance of the dynamic deflection of suspension can the impact of the buffer block under the bad road surface be prevented from causing vehicle damage. Meanwhile, the output control force affects the service life of the suspension, so the fitness function and constraint conditions of cuckoo algorithm are designed in this paper as follows:

$$
\left\{\begin{array}{l}
\min J=\frac{\mathrm{RMS}_{\mathrm{CS}-\mathrm{SMC}}}{\mathrm{RMS}_{\mathrm{SMC}}}, \\
\text { s.t }|U| \leq 1000 N,
\end{array}\right.
$$

where $\mathrm{RMS}_{\mathrm{CS}-\mathrm{SMC}}$ is the root-mean-square value of the suspension dynamic deflection optimized by cuckoo algorithm and $\mathrm{RMS}_{\mathrm{SMC}}$ is the root-mean-square value of the suspension dynamic deflection not optimized. Because the minimum value of the fitness function can ensure that the performance of the optimized controller in the aspect of the dynamic deflection of the suspension is significantly improved, $U$ is the output force of the actuator.

\section{Simulation Results}

The selected parameters of the suspension system and controller are shown in Table 1.

Pavement excitation is selected as grade $\mathrm{C}$ pavement, and the expression is

$$
z_{1}^{\prime}=-2 \pi n_{1} v z(t)+2 \pi n_{0} \sqrt{G\left(n_{0}\right) V} \omega(t) .
$$

The simulation curve of the road surface is shown in Figure 3.

To more intuitively explain the new approach law for active suspension and the superiority of cuckoo algorithm, this section divides the simulation results into two parts for comparative analysis. By substituting the parameters into the matrix, $C B$ is found to be a nonsingular matrix. And the dynamic parameters of the $C$ matrix can be obtained according to equation (17). The parameters of the general approach law are selected by the test method within the optimization range.

4.1. Comparison between the New Approach Law and the Ordinary Sliding Mode Controller. The active suspension is taken as the control object, and the state variable in the new approach law (5) is set as $x_{2}$; that is, the displacement of the car body is taken as the main optimization object, and MATLAB/Simulink is used for simulation. The simulation results are shown in Figures 4-6. 


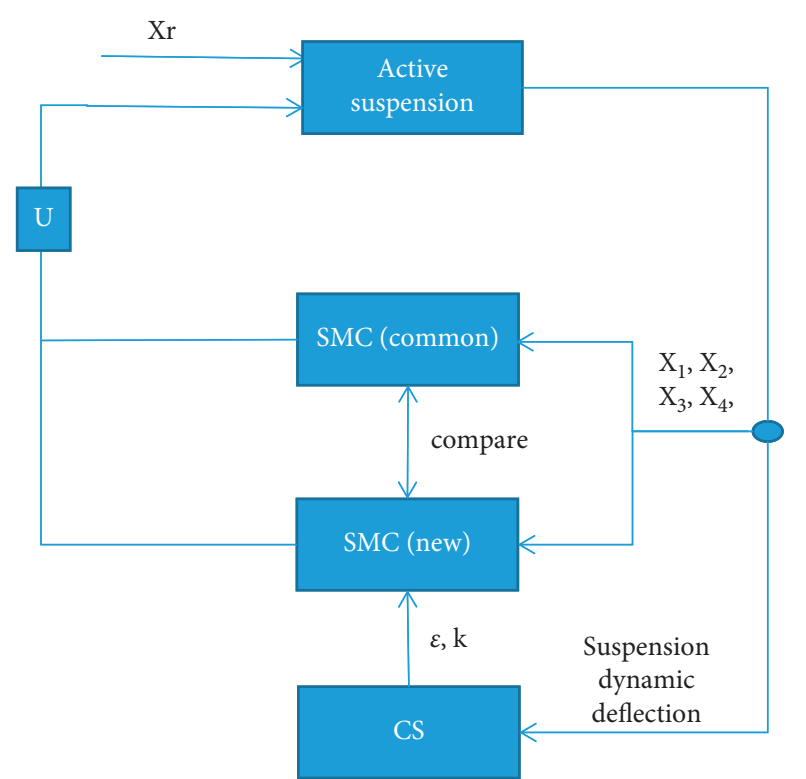

Figure 2: Optimization scheme.

TABLE 1: Suspension parameters.

\begin{tabular}{lccc}
\hline Serial number & Parameter & Unit & Value \\
\hline 1 & $m_{s}$ & $\mathrm{~kg}$ & 320 \\
2 & $m_{t}$ & $\mathrm{~kg}$ & 16 \\
3 & $k_{s}$ & $\mathrm{~N} / \mathrm{m}$ & 33000 \\
4 & $k_{t}$ & $\mathrm{~N} / \mathrm{m}$ & 185000 \\
5 & $c_{s}$ & $\mathrm{Ns} / \mathrm{m}$ & 1013 \\
6 & $v$ & $\mathrm{~m} / \mathrm{s}$ & 10 \\
7 & $p_{a}$ & - & $20 \%$ \\
8 & $\varepsilon_{\mathrm{s}}$ & - & 0.06 \\
9 & $\mathrm{k}_{\mathrm{s}}$ & - & 100 \\
10 & $\mathrm{a}$ & - & 1.1 \\
11 & $\mathrm{c} 1$ & - & -3 \\
12 & $\mathrm{c} 2$ & - & 4 \\
13 & $n$ & - & 20 \\
\hline
\end{tabular}

From the simulation results, it can be found that the sliding mode controller based on the new approach law has an obvious advantage in the aspect of suspension dynamic deflection than the ordinary sliding mode controller. In the body acceleration, the advantage is not obvious. The effect of two sliding mode controllers is almost the same in the dynamic displacement of the tire. Based on the analysis in Figure 3, it can be known that the sliding mode controller based on the new approach law makes the stable rate of the state variable $x_{2}$ faster, and the two performance indexes related to $x_{2}$ in active suspension are the dynamic deflection of suspension and the dynamic displacement of the tire. This shows that the sliding mode controller based on the new approach law still has a good effect in active suspension. In this paper, RMS is used to compare the effect of simulation results. The dynamic deflection of suspension is based on RMS 0.0099 of the new sliding mode approach law. The RMS of the ordinary sliding mode is 0.0103 , and the dynamic deflection performance of suspension is improved by $4 \%$.

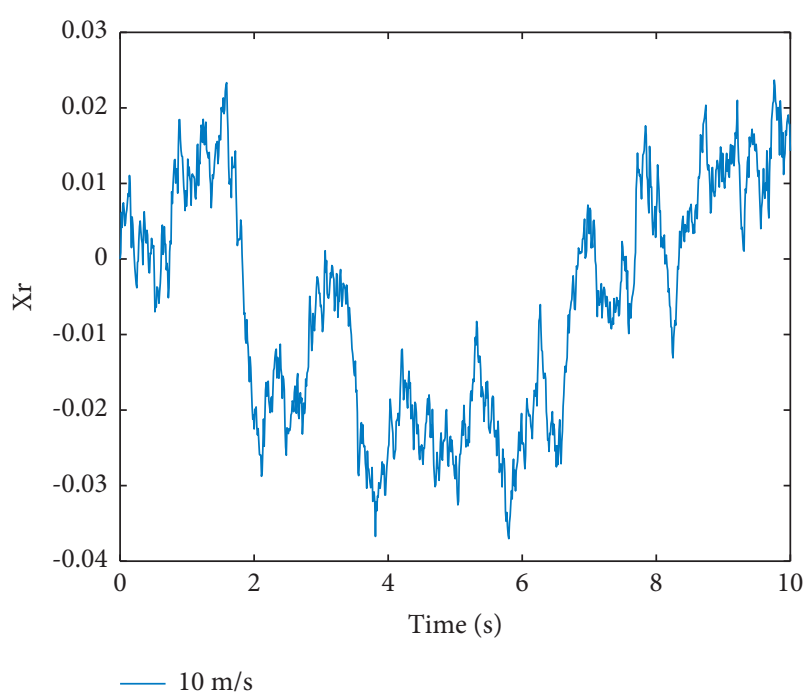

Figure 3: Road excitation.

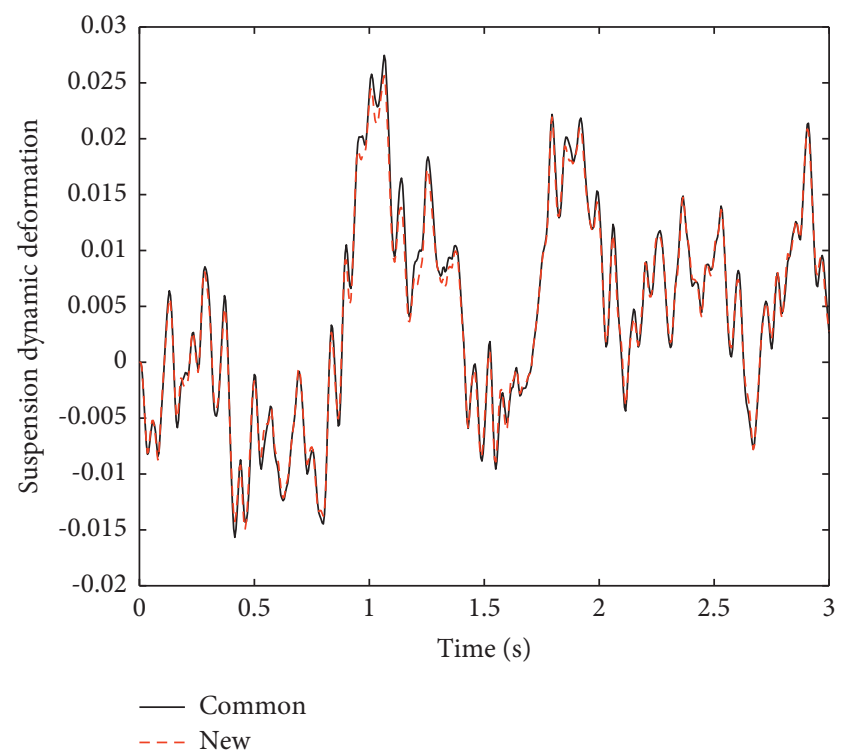

FIgUre 4: Dynamic deflection of suspension.

4.2. Comparison of Cuckoo Search Algorithm Optimization and the Unoptimized Controller. Although based on a new reaching law of sliding mode controller has good performance, in order to achieve the optimal performance of the suspension dynamic deflection, the selection of controller parameters is very important; this paper adopts the cuckoo algorithm based on the sliding mode controller of a new sliding mode reaching law parameters' optimization, according to the design of fitness function, algorithm iterative 200 times, and fitness curves are shown in Figure 7.

When the optimal result is reached, the value of the new approach law parameter $\varepsilon$ is 0.0444 and $k$ is 13.9254 . This paper also provides a comparison with other control methods. The fuzzy controller has the advantages of robustness, adaptability, and fault tolerance. A two-dimensional active suspension fuzzy controller is designed in this 


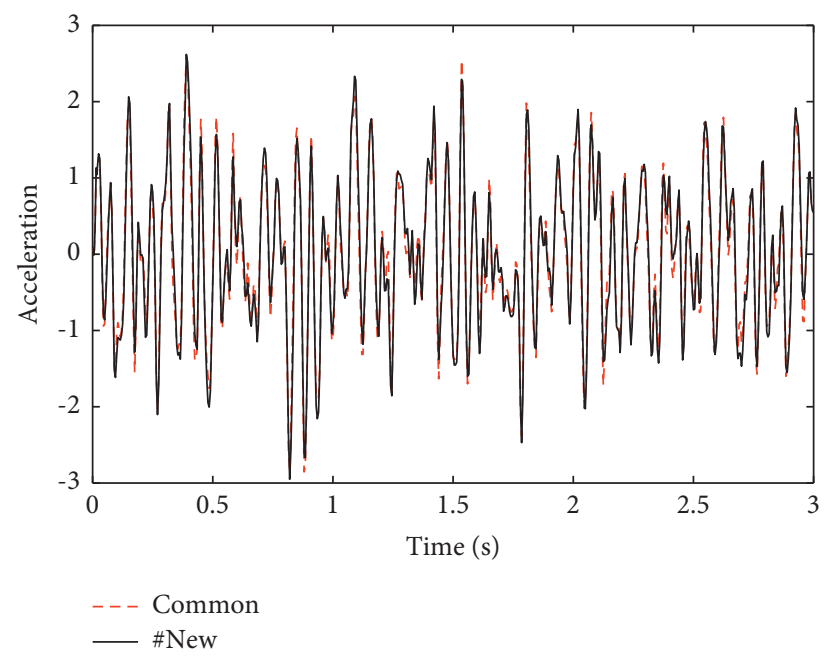

Figure 5: Body acceleration.

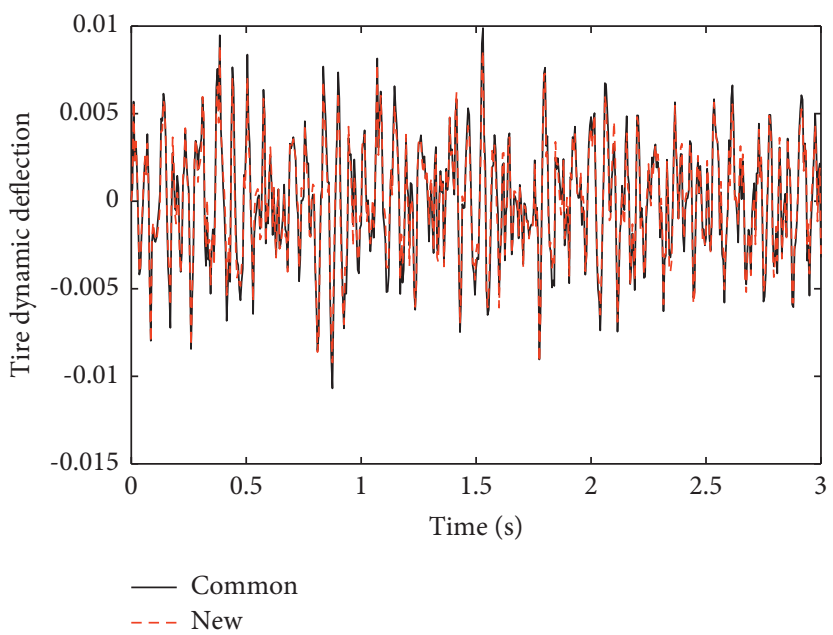

Figure 6: Dynamic displacement of the tire.

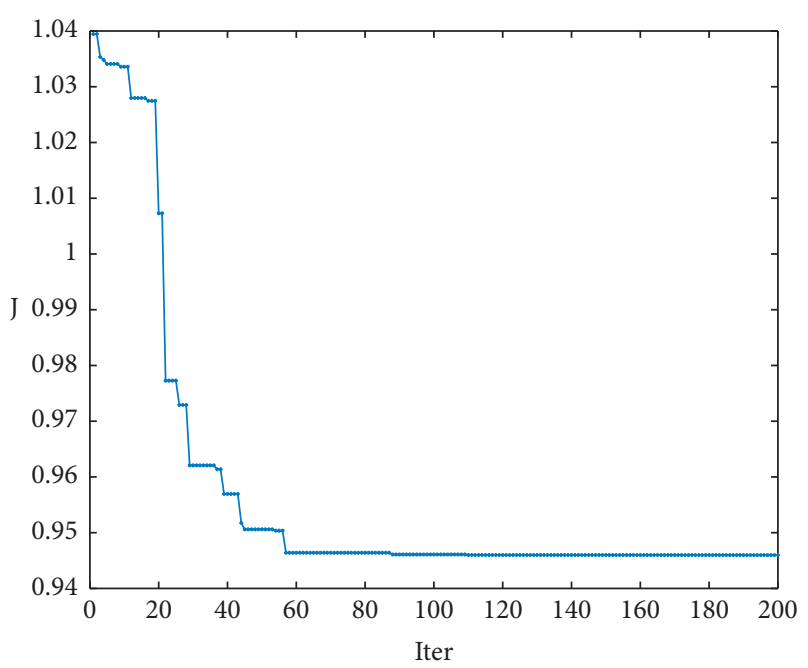

Figure 7: Fitness curve of CS. paper, which takes acceleration and velocity $v$ as the input and force $u$ as the output. $a$ 's universe is $[-8,8], v$ 's universe is $[-1,1], u$ 's universe is $[-1000,1000]$, and fuzzy universe is $[-3,3]$. [NB, NS, Z, PS, PB] is used as the fuzzy language in this paper. The choice of the membership function is the triangle, and the method of eliminating fuzzy is a bifocal method. Fuzzy rules are shown in Table 2.

The three suspension performance indicators are shown in Figures 8-11 shows the output control force of the suspension.

According to the simulation results, it is found that, after the active suspension has a very superior performance index, the cuckoo search algorithm completes the optimization target according to the requirements of the fitness function again; that is to say, within the limit of the output force, the dynamic deflection index of the suspension is optimized to ensure the service life of the active suspension. The sliding mode controller optimized by CS algorithm 
TABLe 2: Fuzzy rules.

\begin{tabular}{lcccc}
\hline$v_{c c} / a_{c c}$ & NB & NS & Z & PS \\
\hline NB & PB & PB & PS & PS \\
NS & PB & PS & PS & Z \\
Z & PS & PS & ZS & NS \\
PS & PS & NS & NS & NS \\
PB & Z & NS & NS & NB \\
\hline
\end{tabular}

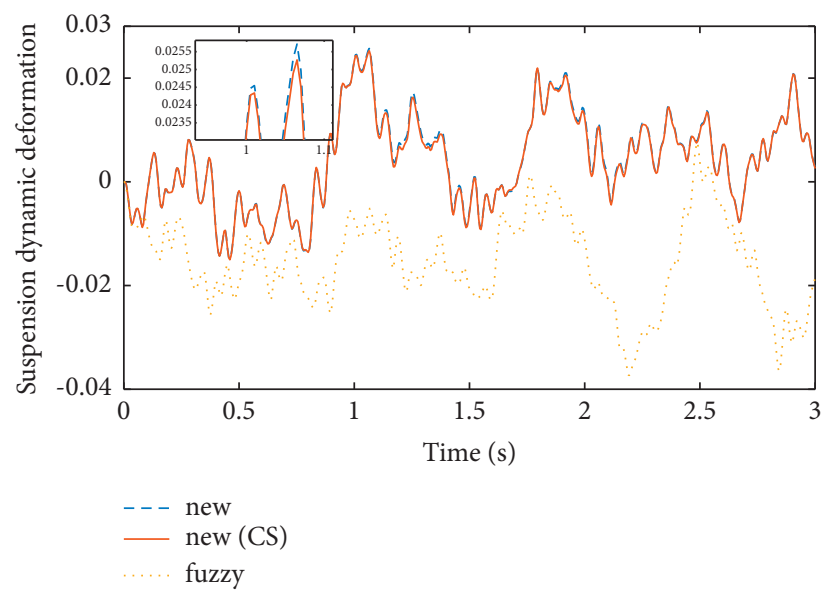

Figure 8: Suspension dynamic deflection 2.

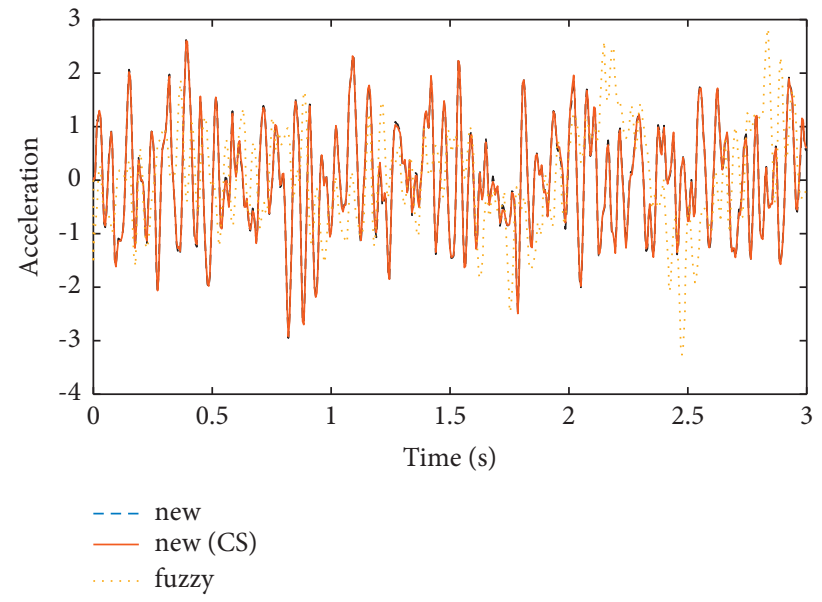

Figure 9: Body acceleration 2.

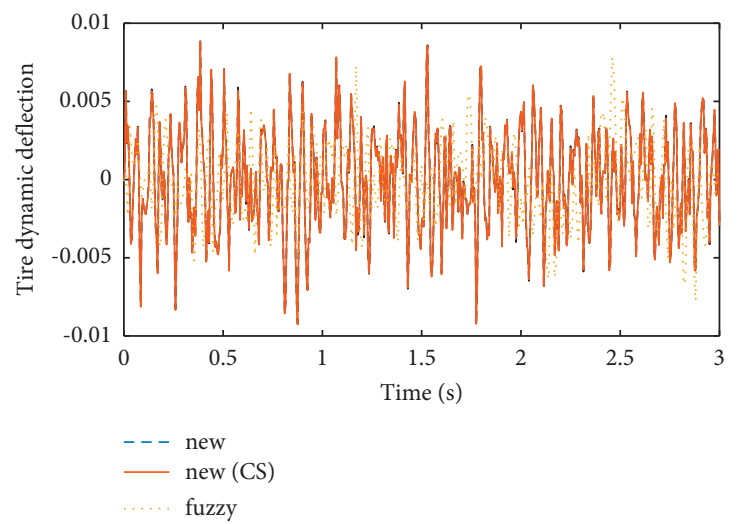

Figure 10: Tire dynamic displacement 2. 


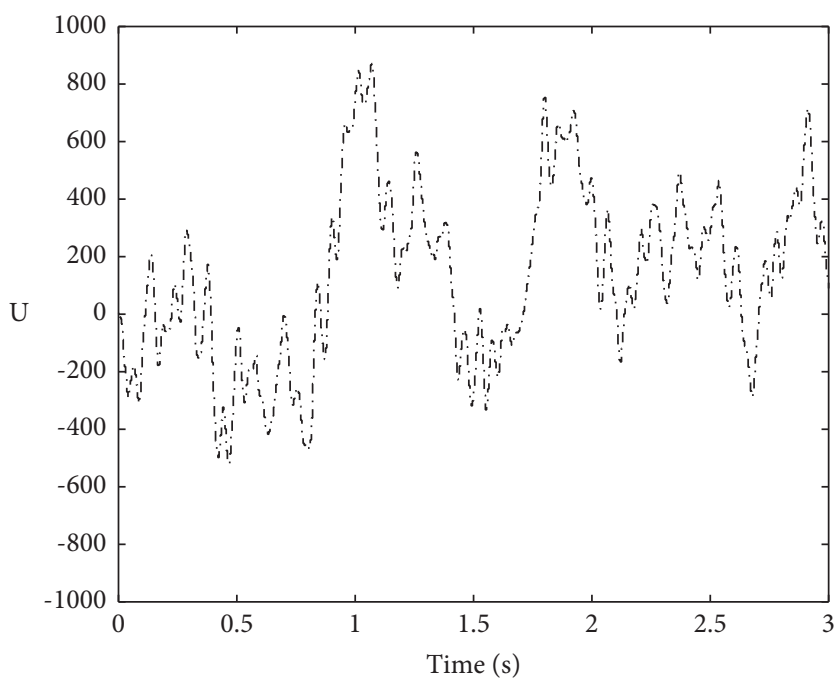

Figure 11: Output force of the controller.

TABLE 3: Simulation suspension optimization results.

\begin{tabular}{lcc}
\hline & RMS (suspension deflection) & Optimization effect \\
\hline General sliding mode & 0.0103 & - \\
New sliding mode & 0.0099 & $4 \%$ \\
Fuzzy control & 0.0134 & $-30 \%$ \\
Optimized sliding mode (CS) & 0.0094 & $8.7 \%$ \\
\hline
\end{tabular}

performs better than the fuzzy controller in acceleration and suspension dynamic deflection. In terms of the tire dynamic displacement index, the effect of the three controllers is very close. The root-mean-square value of the suspension dynamic deflection optimized by these controllers is shown in Table 3.

\section{Conclusions}

In this paper, cuckoo search algorithm is used to optimize the active suspension sliding mode controller which adopts the new exponential reaching law parameters. Compared with the traditional sliding mode controller design scheme, the active suspension control effect is further improved. The feasibility of the optimization scheme is proved. In future studies, more complex suspension models and algorithms with stronger optimization ability can be combined to make the performance of active suspension better and better.

\section{Data Availability}

The raw/processed data required to reproduce these findings cannot be shared at this time as the data also form part of an ongoing study.

\section{Conflicts of Interest}

The authors declare that they have no conflicts of interest.

\section{References}

[1] G. Wang, M. Chadli, H. Chen, and Z. Zhou, "Event-triggered control for active vehicle suspension systems with networkinduced delays," Journal of the Franklin Institute, vol. 356, no. 1, pp. 147-172, 2019.

[2] H. Pan and W. Sun, "Nonlinear output feedback finite-time control for vehicle active suspension systems," IEEE Transactions on Industrial Informatics, vol. 15, no. 4, pp. 2073-2082, 2019.

[3] F. Lai and B. Hu, "Research status of automotive active suspension technology," Journal of Nanjing University of Science and Technology, vol. 43, no. 4, pp. 518-526, 2019.

[4] R. Wang, H. Jing, F. Yan, H. Reza Karimi, and N. Chen, "Optimization and finite-frequency $\mathrm{H} \infty$ control of active suspensions in in-wheel motor driven electric ground vehicles," Journal of the Franklin Institute, vol. 352, no. 2, pp. 468-484, 2015.

[5] H. Pan, H. Li, W. Sun, and Z. Wang, "adaptive fault-tolerant compensation control and its application to nonlinear suspension systems," IEEE Transactions on Systems, Man, and Cybernetics: Systems, vol. 50, no. 5, pp. 1766-1776, 2020.

[6] Y. Wei, Y. Wang, C. K. Ahn, and D. Duan, "IBLF-based finitetime adaptive fuzzy output-feedback control for uncertain MIMO nonlinear state-constrained systems," IEEE Transactions on Fuzzy Systems, 2020.

[7] W. Zhou, Y. Wang, and C. K. Ahn, "Adaptive fuzzy backstepping-based formation control of unmanned surface vehicles with unknown model nonlinearity and actuator saturation," IEEE Transactions on Vehicular Technology, vol. 69,2020

[8] H. Li, H. Liu, S. Hand, and C. Hilton, "Design of robustHocontroller for a half-vehicle active suspension 
system with input delay," International Journal of Systems Science, vol. 44, no. 4, pp. 625-640, 2013.

[9] J. Mrazgua, E. H. Tisssir, and M. Ouahi, "Fuzzy fault-tolerant Ho control approach for nonlinear active suspension systems with actuator failure," Procedia Computer Science, vol. 148, pp. 465-474, 2019.

[10] K. Karim Afshar, A. Javadi, and M. R. Jahed-Motlagh, "Robust control of an active suspension system with actuator time delay by predictor feedback," IET Control Theory \& Applications, vol. 12, no. 7, pp. 1012-1023, 2018.

[11] H. Jing, R. Wang, C. Li, and J. Bao, "Robust finite-frequency H control of full-car active suspension," Journal of Sound and Vibration, vol. 441, pp. 221-239, 2019.

[12] C. Pan, J. Rong, D. Xiang, and Y. Zheng, "Study on ride comfort of active suspension with 9 degrees of freedom," Transactions of Beijing Institute of Technology, vol. 39, no. 10, pp. 1012-1017, 2019.

[13] L. I. Nkomo, A. Dove, M. T. Ngwako, and O. T. Nyandoro, "Heaviside based optimal control for ride comfort and actuation energy optimisation in half-car suspension systems," IFAC-PapersOnLine, vol. 50, no. 2, pp. 259-264, 2017.

[14] S.-A. Chen, J.-C. Wang, M. Yao, and Y.-B. Kim, "Improved optimal sliding mode control for a non-linear vehicle active suspension system," Journal of Sound and Vibration, vol. 395, pp. 1-25, 2017.

[15] Y. Chen and Y. Shao, "Research on optimal control of vehicle active suspension system," no. 8, pp. 47-52, 2018.

[16] C. Y. Tang, G. Y. Zhao, and Y. M. Zhang, "Research on active suspension system based on bp and rbf network algorithm," Advanced Materials Research, vol. 230, pp. 149-153, 2011.

[17] J. Zhang, Y. Shi, and H. Feng, "Research on active suspension control based on bp neural network fuzzy pid Manufacturing Automation," vol. 41, no. 2, p. 3, 2019.

[18] Y. Liu, Q. Zeng, S. Tong, C. L. P. Chen, and L. Liu, “Adaptive neural network control for active suspension systems with time-varying vertical displacement and speed constraints," IEEE Transactions on Industrial Electronics, vol. 66, no. 12, pp. 9458-9466, 2019.

[19] F. Zhao, S. S. Ge, F. Tu, Y. Qin, and M. Dong, "Adaptive neural network control for active suspension system with actuator saturation," IET Control Theory \& Applications, vol. 10, no. 14, pp. 1696-1705, 2016.

[20] Y.-X. Wang, Y.-M. Cai, J. Wang, M. Yao, and S.-A. Chen, "Design method of active suspension lqg controller for rolling motion safety of vehicle," IET Control Theory \& Applications, vol. 5, pp. 138-148, 2017.

[21] K. Sim, H. Lee, J. W. Yoon, C. Choi, and S.-H. Hwang, "Effectiveness evaluation of hydro-pneumatic and semi-active cab suspension for the improvement of ride comfort of agricultural tractors," Journal of Terramechanics, vol. 69, pp. 23-32, 2017.

[22] Z. Ma, Q. Fang, Z. Wu, and H. Liang, "Research on stiffness adjustment preview algorithm of electronic control air suspension system," Mechanical Science and Technology, vol. 38, no. 3, pp. 398-403, 2019.

[23] U. S. Pusadkar, S. D. Chaudhari, P. Shendge, and S. Phadke, "Linear disturbance observer based sliding mode control for active suspension systems with non-ideal actuator," Journal of Sound and Vibration, vol. 442, pp. 428-444, 2019.

[24] B. Jiang, H. R. Karimi, Y. Kao, and C. Gao, “Takagi-sugeno model-based event-triggered fuzzy sliding-mode control of networked control systems with semi-markovian switchings," IEEE Transactions on Fuzzy Systems, vol. 28, no. 4, pp. 673$683,2020$.
[25] L. A. Zhen, B. Hrk, and A. Jy, "Passivity-based robust sliding mode synthesis for uncertain delayed stochastic systems via state observer," Automatica, vol. 111, 2020.

[26] H. Benariba, L. Baghli, and A. Boumediene, "Vertical displacement sliding mode control of a half-vehicle active suspension," in Proceedings of the 2018 5th International Symposium on Environment-Friendly Energies and Applications (EFEA), pp. 1-6, Rome, Italy, September 2018.

[27] B. Jiang and H. R. Karimi, "Observer-based adaptive sliding mode control for nonlinear stochastic markov jump systems via T-S fuzzy modelling: applications to robot arm model," IEEE Transactions on Industrial Electronics, vol. 99, p. 1, 2020.

[28] J. Wang, C. Yang, H. Shen, J. Cao, and L. Rutkowski, "Slidingmode control for slow-sampling singularly perturbed systems subject to markov jump parameters," IEEE Transactions on Systems, Man, and Cybernetics: Systems, 2020.

[29] Y. Yang, F. Chen, and J. Lang, "Sliding mode control of persistent dwell-time switched systems with random data dropouts," Applied Mathematics and Computation, vol. 400, no. 5, Article ID 126087, 2021.

[30] A. Khoudiri, K. Guesmi, and D. Mahi, "Optimized sliding mode control of DC-DC boost converter for photovoltaic system," in Recent Advances in Electrical Engineering and Control Applications, Ser. Lecture Notes in Electrical Engineering, M. Chadli, S. Bououden, and I. Zelinka, Eds., pp. 393-406, Springer International Publishing, Berline, Germany, 2017.

[31] M. Sun, "Sliding mode control parameter tuning based on particle swarm optimization," Mechanical Engineering and Automation, vol. 1, pp. 173-175, 2019.

[32] K. Gao, Z. Guo, Y. Yu, H. Yu, and Y. Zhang, "Design and optimization of sliding mode controller for active suspension of heavy vehicle," Journal of Shandong University of Technology, vol. 34, no. 4, pp. 30-36, 2020.

[33] X. Zhang, K. Zhao, L. Sun, and A. Quntao, "Dynamic quality control of permanent magnet synchronous motor sliding mode variable structure speed control system," vol. 31, no. 15, pp. 47-52, 2011. 